\title{
Feasibility Study on an On-board Dosimeter for a Drone by Image Processing Technology
}

\author{
Yu Terajima ${ }^{\mathrm{a}}$, Hideki Tenzou ${ }^{\mathrm{b}}$, Junya Kuroda ${ }^{\mathrm{a}}$, and Taiki Mimoto ${ }^{\mathrm{a}}$ \\ ${ }^{a}$ Advanced Course in Electronics, Information and Communication Engineering, Electronic System Course, National \\ Institute of technology, Kagawa College, 551 Koda, Takuma-cho, Mitoyo, kagawa 769-1192, Japan \\ ${ }^{b}$ Department of Electronic System Engineering, National Institute of technology, Kagawa College, 551 Koda, Takuma-cho, \\ Mitoyo, kagawa 769-1192, Japan
}

*Corresponding Author: a16510@ sr.kagawa-nct.ac.jp

\begin{abstract}
The purpose of this study was to confirm to have relationship between dose rate in the air and the trajectories generated by $\gamma$-rays in a compact cloud chamber by image difference processing method. After the Fukushima Daiichi Nuclear disaster, dose rate for decontamination area have been monitoring by using a heavy $\gamma$-ray detector on a drone. A cloud chamber has been used to observe the trajectory for charged particles. A new method to monitor dose rate over the decontamination area was proposed in this paper by imaging procedure for trajectories of Compton electrons generated by incident $\gamma$-rays in a light cloud chamber loaded on a drone. Cloud chamber has good feature as a mounted type detector on a drone that the system is constructible to be light, sensitive to low does rate, and has electrical noise tolerance. A compact cloud chamber was built and the chamber was demonstrated for a Cesium 137 $\gamma$-rays source. There was a relationship between dose rate in the air and the number of visible trajectories by a new image processing for the trajectories of Compton electrons. As a result, it was found that the compact cloud chamber have a good feature for air monitoring system mounted on a drone, It was also confirmed that incident direction of gamma rays can be estimated by using image processing technology.
\end{abstract}

Keywords: cloud chamber, drone, image processing.

\section{Introduction}

The purpose of this study was to investigate a relationship between dose rate in the air and the trajectories generated by $\gamma$-rays in a compact cloud chamber by image difference processing method. After Fukushima Daiichi Nuclear disaster, nuclear decontamination effect has been monitored by a drone loaded a radiation detector. It's important to have multiple plans of nuclear decontamination across the ages because the dose rate of the decontamination area is changing by decay of nuclear material, rain, or wind after the decontamination. JAEA are monitoring by using a small air craft loaded radiation detector ${ }^{(1)}$. This method has advantage to monitor the decontamination effect in terms of more wide area, much faster than one of ground-based observation.

A drone is often used to capture images for wide area from high in the skies in the terms of saving the initial cost and running cost nowadays. The camera loaded on the drone is enough light to be carried. A drone, however, doesn't have enough power to carry a heavy scintillation detector. It's important to develop a light dosimeter. A cloud chamber is one of light detectors, although it has been used to observe the trajectory for charged particles. Therefore, a new method to monitor dose rate over the decontamination area was proposed in this paper by imaging procedure for trajectories of Compton electrons generated by incident $\gamma$-rays in a light cloud chamber loaded on a drone. A cloud chamber has good feature as a mounted type detector on a drone that the system is constructible to be light, sensitive to low does rate, and has an electrical noise tolerance. In this paper, a relationship between dose rate in the air and number of visible trajectories generated by $\gamma$-rays was investigated by using a new method of image processing. Constant time of the compact cloud chamber in measuring the dose rate was also surveyed. 


\section{Overview}

\subsection{Compact Cloud Chamber}

A compact cloud chamber was built to survey relationship between dose rate in the air and number of visible trajectories by using a new method of image processing. Fig.1 shows an overview of the system to record the live images of trajectories generated by incident $\gamma$-rays in the cloud chamber. The system is composed of the compact cloud chamber attached a high-voltage source and a USB camera. A USB camera is connected to a conventional Windows PC to record the live images in 30 FPS. The high-voltage of $2 \mathrm{kV}$ is applied to clear the chamber of unwanted ions. We can see typical trajectories created by Compton electrons scattered multiple times in cloud chamber. In order to remove static light noise, a threshold is applied to the difference image such that only large differences in intensity remain in the difference image between each image frame. After the difference procedure, the number of white pixel $\mathrm{N}$ was counted. The total weight of the system including a battery is $363 \mathrm{~g}$. A payload of DJI S900 which is a conventional commercially-available drone is $8.4 \mathrm{~kg}$ so that the system is carried without serious affects the performance of flight if the center of mass of the loaded system is adjusted.

\subsection{Image Processing Program}

Fig.2 is flowchart of image processing program. The image processing program consist of a part to generate a differential image and part to a count pixels. As a first step, the program reads trajectory data from an image captured in cloud chamber. As a next step, RGB value on each pixels in image is compared the $(\mathrm{N})$ th image with $(\mathrm{N}+1)$ th image. If the difference of RGB date is larger than a threshold, the number is recognized as differential pixel and is counted. Fig.3-(a) and (b) are image pre differential processing, and Fig.3-(c) is the result of differential image.

\section{EXPERIMENT}

The system was demonstrated to survey under environment of dose rate range from about $2 \mu \mathrm{Sv} / \mathrm{h}$ to 7 $\mu \mathrm{Sv} / \mathrm{h} .1 \mathrm{~kg}$ of dry ice was used to cool down the bottom of the cloud chamber to about $-40^{\circ}$. Cesium 137 was used as a $\gamma$-rays source of $511 \mathrm{keV}$. A dose rate at the surface of the cloud chamber was changed by the distance between cloud chamber and the $\gamma$-rays sources and was measured by a $\gamma$-ray survey meter ALOKA TCS-172. We can see typical trajectories created by Compton electrons scattered in a cloud chamber are shown inner a circle with red line in Fig.5. The constant time of the cloud chamber was measured by changing a number of frames to count a white pixel in each frame. The changed numbers of frames correspond to from 1, 5, 30, and $60 \mathrm{sec}$.

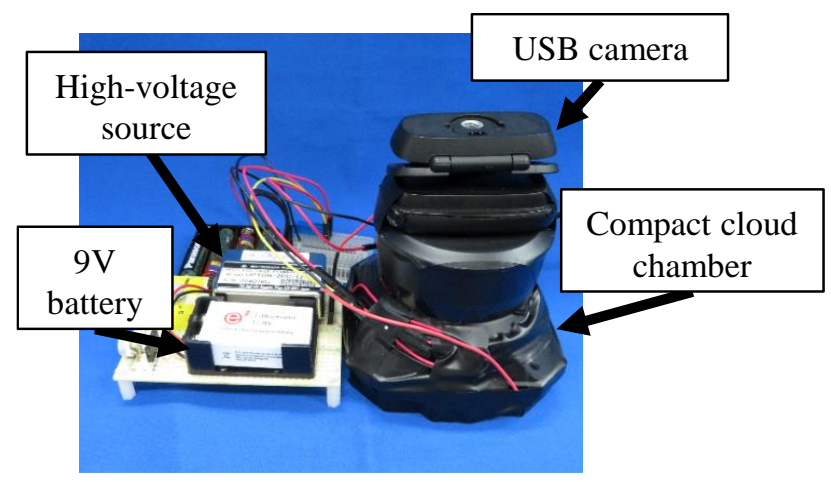

Fig. 1. Overview of the system to recode the live image of trajectories.

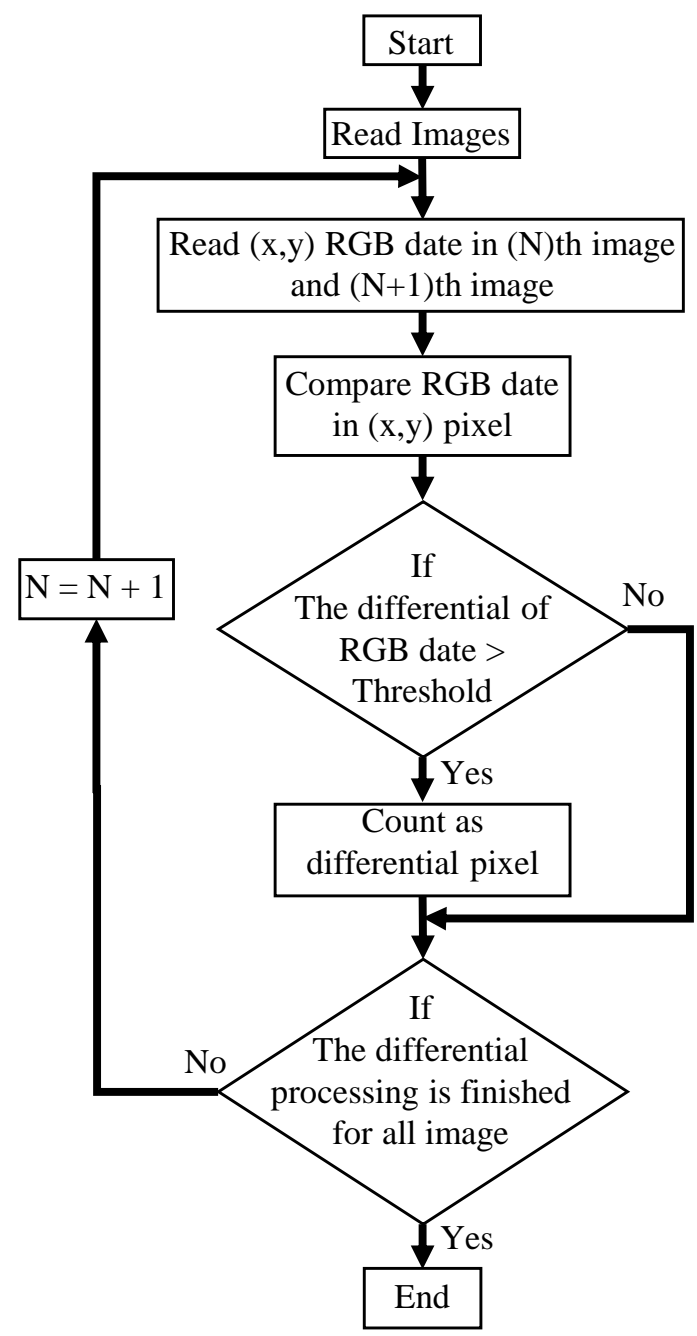

Fig. 2. Flowchart of image processing program. 


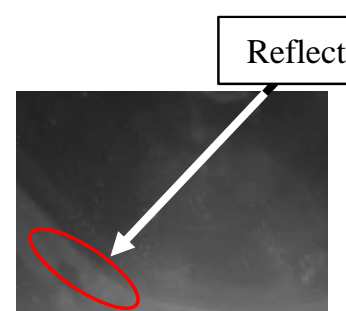

(a) (N)th image. (b) $(\mathrm{N}+1)$ th image.

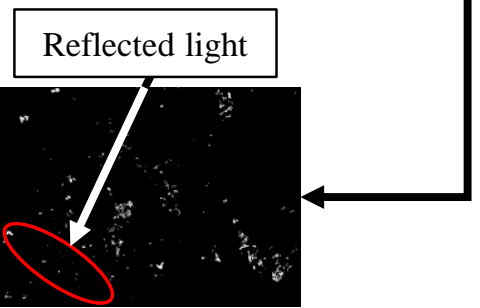

(c) Differential image $(\mathrm{N}-(\mathrm{N}-1))$.

Fig. 3. Process of image differential processing.

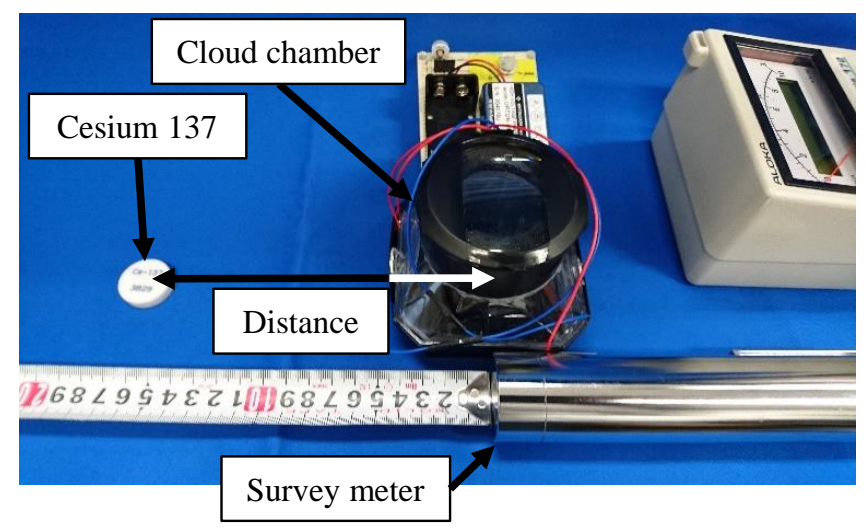

Fig. 4. Experiment to survey the relationship between dosage and number of trajectories.

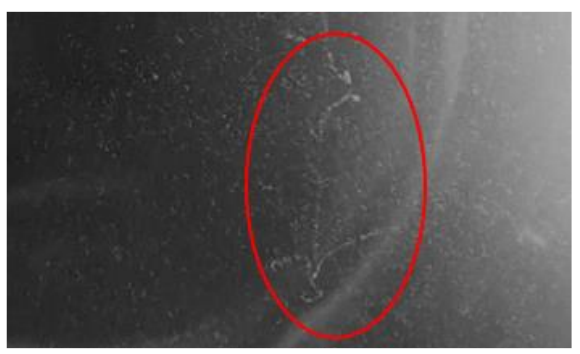

Fig. 5. Trajectories of electrons by Compton scattering.

\section{RESULT}

\subsection{Relationship between Dosage and Number of Trajectories}

The typical images captured by the USB camera is listed in Fig. 7 when dose rate is changed from $2.6 \mu \mathrm{Sv} / \mathrm{h}$ to $7.3 \mu \mathrm{Sv} / \mathrm{h}$ proportional to the distance from the surface of the cloud chamber. Each frame image is taken the difference between the image of the moment and previous frame image in order to remove the light noise. The typical difference image is shown in Fig.7. The values of dose rate in Fig.7 are background, $2.60 \mu \mathrm{Sv} / \mathrm{h}, 3.77 \mu \mathrm{Sv} / \mathrm{h}$ and 7.30 $\mu \mathrm{Sv} / \mathrm{h}$ on Fig.7-(a), (b), (c), and (d), respectively. The total number of trajectories in all 1800 image frames $(60 \mathrm{sec})$ reveals a correlation with each dose rate, as the relationship is plotted in Fig.8. Linearity is confirmed between the dose rate and the trajectories in Fig.8.

\subsection{Constant Time to Measure Dose Rate over 2.6 $\mu \mathrm{Sv} / \mathrm{h}$}

The summarized number of image frame is changed for each dose rate to estimate minimal constant time to measure dose rate over $2.6 \mu \mathrm{Sv} / \mathrm{h}$. To normalize the summarized number of white pixel for each constant time, the summarized number is derived from the total count at $30 \mathrm{sec}$ of time constant for each dose rate and the number is called normalized number. There is a strong correlation between dose rate and time constant as is shown in Fig. 9. We can also see the difference of count ratio from the value at time constant of $60 \mathrm{sec}$. Time constant correspond to within the $10 \%$ tolerance was over $5 \mathrm{sec}$ of time constant from Fig. 9.

\subsection{Relationship between Direction and Trajectories}

It was supposed that the system has the ability to find the directions of incident $\gamma$-rays. A $6 \mathrm{~mm}$ thickness lead plate is set at the center of cloud chamber in order to survey whither the direction of radiation source is estimated by the compact cloud chamber as shown in Fig. 10. The position of radiation source is placed the plate vertically for the incident gamma rays in the demonstration. Fig. 10-(a) and (b) are the images for $\gamma$-rays source are placed near the cloud chamber. We can see the number of trajectories are different in each area.

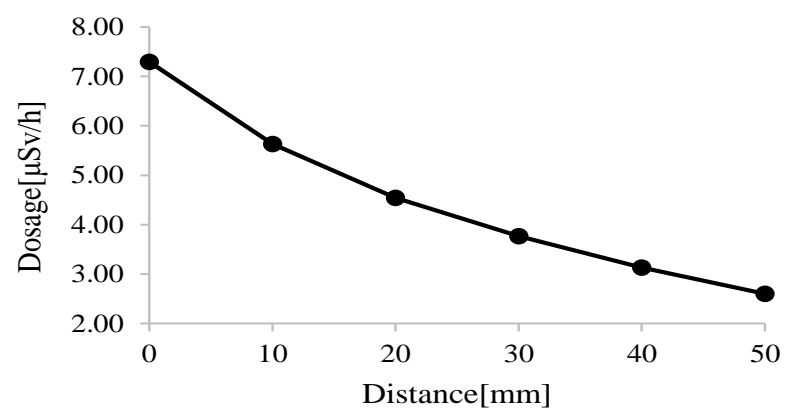

Fig. 6. Relationship between dosage and distance from a radiation source. 


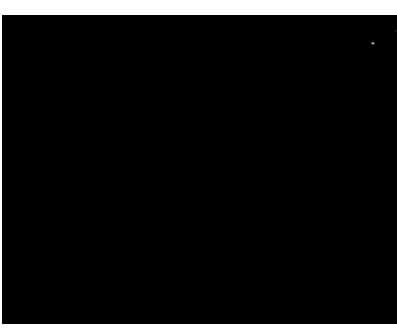

(a) Background.

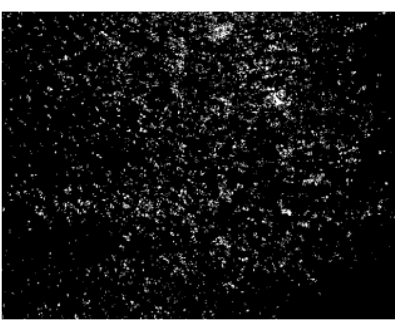

(c) $3.77 \mu \mathrm{Sv} / \mathrm{h}$.

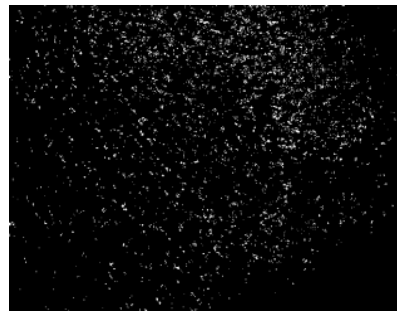

(b) $2.60 \mu \mathrm{Sv} / \mathrm{h}$.

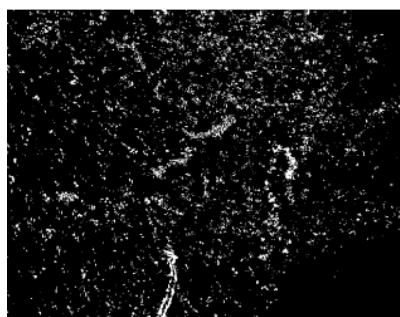

(d) $5.63 \mu \mathrm{Sv} / \mathrm{h}$
Fig. 7. Differential processing image under background, $2.60 \mu \mathrm{Sv} / \mathrm{h}, 3.77 \mu \mathrm{Sv} / \mathrm{h}$, and $7.30 \mu \mathrm{Sv} / \mathrm{h}$.

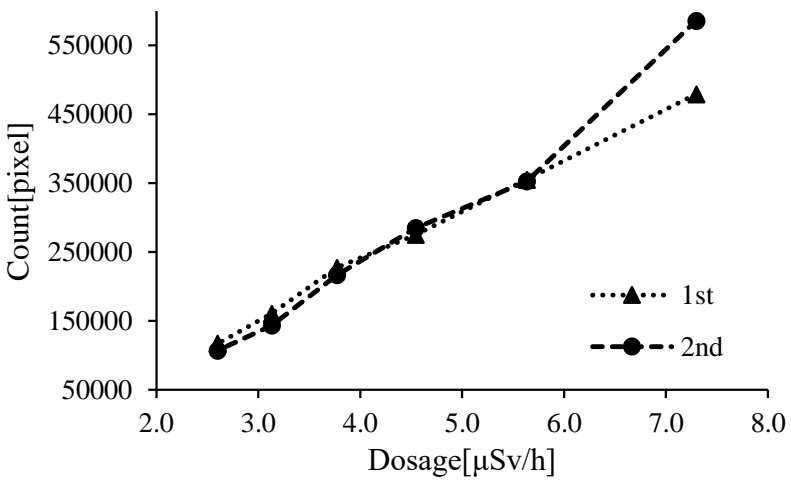

Fig. 8. Relationship between dosage and count ratio.

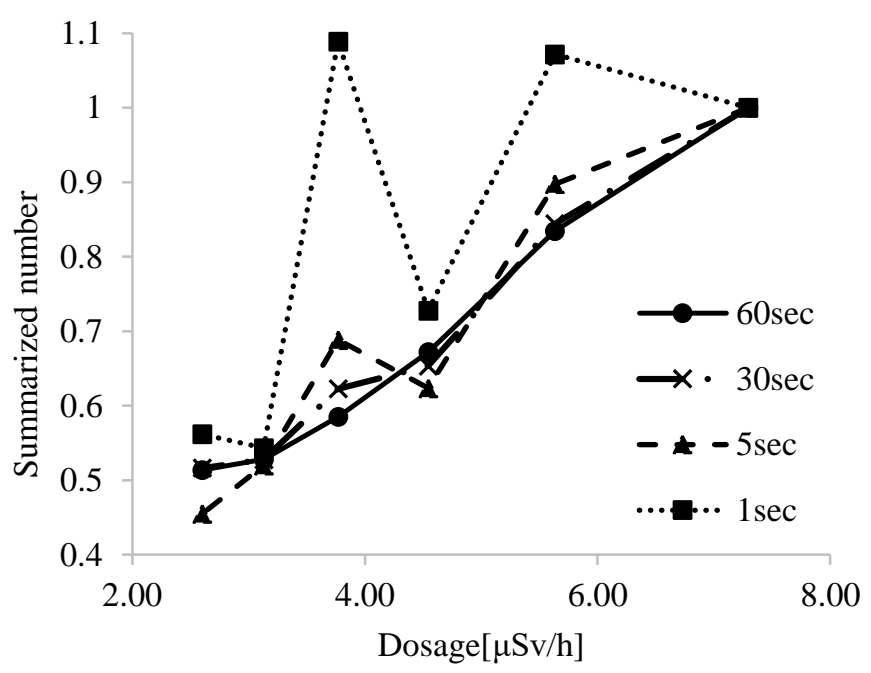

Fig. 9. Relationship between count ratio and measurement time at 30 FPS.

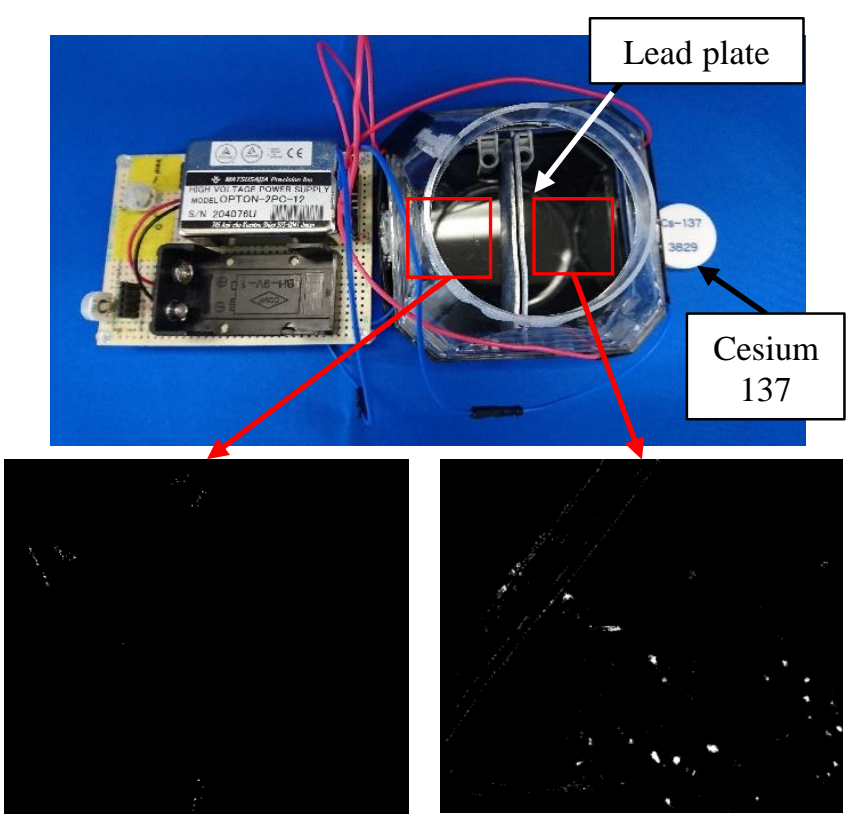

(a) Shielded side.

(b) Radiation source side

Fig. 10. Comparison shielded side with radiation sources side.

\section{SUMMARY}

A light cloud chamber which will be loaded on a drone was built to monitor an effect of nuclear decontamination for large area. The weight of chamber was 363g. Trajectories of Compton electrons generated by incident $\gamma$-rays from Cesium 137 were observed in the chamber and analyzed by image difference processing method. Dose rate response of the chamber for $\gamma$-rays was surveyed from $2.6 \mu \mathrm{Sv} / \mathrm{h}$ to $7.3 \mu \mathrm{Sv} / \mathrm{h}$. As a result, it was confirmed that there was linearity between the dose rate in the air and the trajectories generated by $\gamma$-rays in a compact cloud chamber. It was also confirmed that incident direction of gamma rays can be estimated by using image processing technology. The constant time to measure the dose rate is over $5 \mathrm{sec}$ within $10 \%$ tolerance. It was found that a compact cloud chamber have a good feature for air monitoring system on a drone.

\section{References}

(1) Torii Tatsuo, Sanada Yukihisa, Sugita Takeshi, Kondo Atsuya, Shikaze Yoshiaki, Takahashi Masaki, Ishida Mutsushi, Nishizawa Yukiyasu, and Urabe Yoshimi : "Investigation of Radionuclide Distribution Using aircraft for Surrounding Environmental Survey from Fukushima Dai-ichi Nuclear Power plant", JAEA-Technology 2012-036, Vol. 184, pp.66-68, 2012 